\title{
ON THE TOPOLOGICAL STRUCTURE OF THE FERMAT SURFACE OF DEGREE 5
}

\author{
By Yukio Matsumoto
}

\begin{abstract}
We will give the monodromy representation of a certain fibration of the Fermat surface of degree 5 explicitly in terms of Dehn twists about concrete curves. This paper is a sequel to Ahara's work [1]
\end{abstract}

\section{Introduction}

Let $V_{5}$ be the complex projective hypersurface in $C P_{3}$ defined by the equation of degree 5

$$
z_{0}^{5}-z_{1}^{5}=z_{2}^{5}-z_{3}^{5}
$$

where $z_{0}, z_{1}, z_{2}, z_{3}$ are the homogenuous coordinates. From topological viewpoint, this surface is a simply-connected 4-manifold. The Euler characteristic and the signature of $V_{5}$ are equal to 55 and -35 , respectively (see [5]). By Freedman [4] $V_{5}$ is homeomorphic to $9 C P_{2} \# 44 \overline{C P}_{2}$, but by Donaldson [3] it is not diffeomorphic to this connected sum.

Our motive is to understand the topological structure of $V_{5}$ through a holomorphic fibration over the Riemann sphere $C P_{1}=C \cup\{\infty\}$.

The fibration $f: V_{5} \rightarrow C P_{1}$ is defined as follows:

$$
f:\left[z_{0}, z_{1}, z_{2}, z_{3}\right] \mapsto \begin{cases}z_{2}^{4} / z_{0}^{4}, & \text { if } z_{0}=z_{1} \text { and } z_{2}=z_{3} \\ \left(z_{0}-z_{1}\right) /\left(z_{2}-z_{3}\right), & \text { otherwise. }\end{cases}
$$

A general fiber of this fibration is a Riemann surface of genus 3. In unpublished notes (1990), the author determined the positions and the topological types of all singular fibers in $f: V_{5} \rightarrow C P_{1}$; let $F_{\sigma}$ denote the fiber over a point $\sigma \in C P_{1}$. Then $F_{\sigma}$ is a singular fiber if and only if $\sigma$ belongs to the following set $S F$ consisting of 17 points:

$$
S F=\left\{\sigma \mid \sigma^{5}=-1 / 4,1, \text { or }-4\right\} \cup\{0, \infty\} .
$$

If $\sigma=0$ or $\infty, F_{\sigma}$ is a union of 4 complex lines meeting in a point. If $\sigma$ is a 5 -th root of $1, F_{\sigma}$ is a union of two complex lines and a conic, meeting in 5 points transversely. If $\sigma$ is a 5 -th root of $-1 / 4$ or of $-4, F_{\sigma}$ is an irreducible stable curve of virtual genus 3

1991 Mathematics Subject Classfication. Primary 57M50, Secondary 32J15

This paper is dedicated to the memory of Professor Yukiyosi Kawada

Received October 30, 1993. 
which has two transverse sef-intersection points. See the table below.

\begin{tabular}{|l|c|c|c|}
\hline & $\sigma=0$ or $\infty$ & $\sigma^{5}=1$ & $\sigma^{5}=-\frac{1}{4}$ or -4 \\
\hline$F_{\sigma}$ & &
\end{tabular}

Remark. In the same unpublished notes, the author determined the positions and the types of all singular fibers appearing in a similar fibration of the Fermat surface of general degree $n(n \geq 2)$. These results and the methods are summarized in $[1$, Section 2].

To accomplish the topological description of the fibration $f: V_{5} \rightarrow C P_{1}$, it suffices to give the monodromy representation

$$
\rho: \pi_{1}\left(C P_{1}-S F, \sigma_{0}\right) \rightarrow \mathcal{M}_{3}
$$

where $\mathcal{M}_{3}$ is the mapping class group of a closed oriented surface of genus 3 , and $\sigma_{0}$ is a base point chosen in $C P_{1}-S F$.

Ahara [1] has essentially determined $\rho$. He presents a general fiber $F_{\sigma}$ as a 4-fold irregualar branched covering of $C P_{1}$ branched at 8 points. By numerical analysis using a computer, he has described motions of the branch points induced by movements of $\sigma$ along paths in $C P_{1}-S F$. He has also given an algorithm to compute the action of the monodromy on the fundamental group of $F_{\sigma_{0}}$ up to inner automorphisms. Thus by Dehn-Nielsen's theorem [7] which states that $\operatorname{Aut}\left(\pi_{1} F_{\sigma_{0}}\right) / \operatorname{Inn}\left(\pi_{1} F_{\sigma_{0}}\right) \cong \mathcal{M}_{3}$, it is possible in principle to describe the monodromy representation $\rho$ explicitly in terms of Dehn twists. However, this last step remains undone in Ahara's paper. To carry it out actually is easy for some loops as Ahara asserts in Remark 4 of [1, Section 1], but for general loops it is not so immediate.

The purpose of this paper is to complete this last step ; we will take Ahara's computaion as a starting point and will give the monodromy representation $\rho$ explicitly in terms of Dehn twists about concrete simple closed curves on $F_{\sigma_{0}}$ (Theorem 2.1). Our method does not depend on Ahara's algorithm and is more pictorial.

\section{Main results}

Following Ahara [1], we choose $\sigma_{0}=11 / 10$ as a base point of $C P_{1}-S F$. The fiber $F_{\sigma_{0}}$ is presented as a 4 -fold irregular branched covering of $C P_{1}$ branched at 8 points $\Sigma=$ $\{A, \bar{A}, B, \bar{B}, C, \bar{C}, D, \bar{D}\}$, where $A \approx-0.9256+0.3786 \sqrt{-1}, B \approx-0.3800+0.9250 \sqrt{-1}$ $, C \approx 0.2159+0.9764 \sqrt{-1}, D \approx 0.3246+0.9458 \sqrt{-1}$. See Ahara [1, Section 4]. 


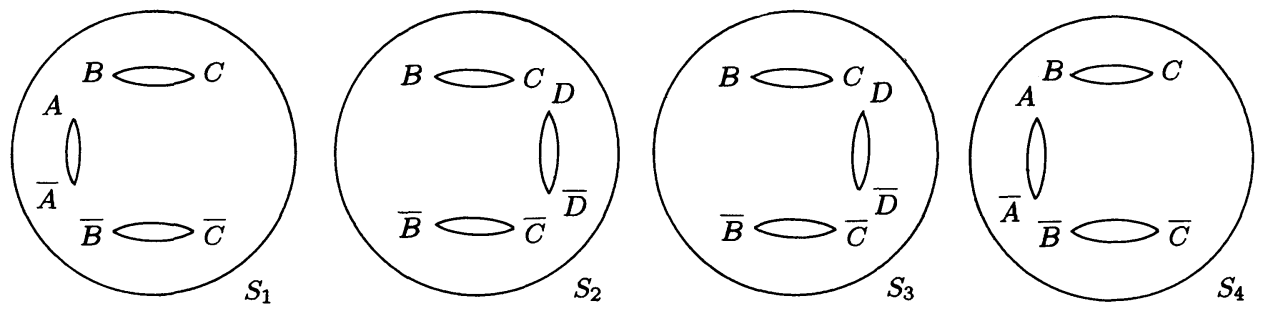

Figure 1
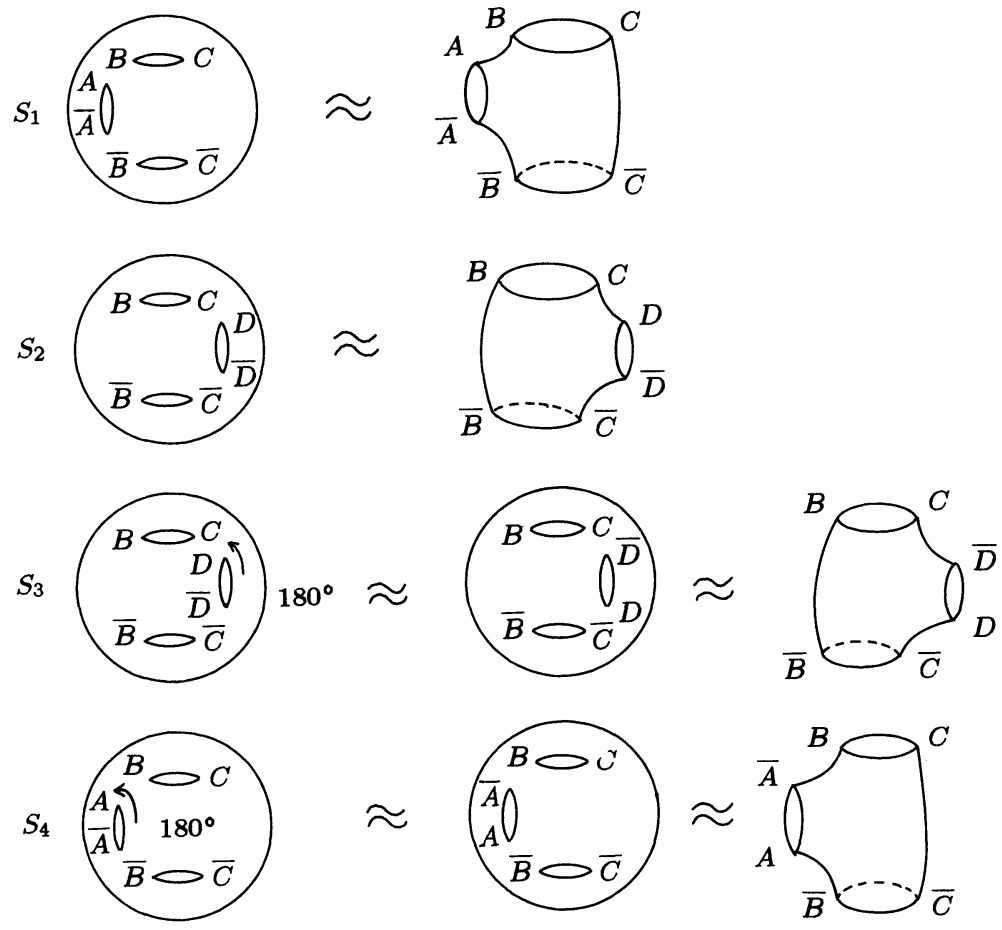

Figure 2

The branching monodromy $\xi: \pi_{1}\left(C P_{1}-\Sigma, 0\right) \rightarrow \mathfrak{S}_{4}$ associated with the branched covering $F_{\sigma_{0}} \rightarrow C P_{1}$ is computed as follows:

$$
\begin{aligned}
& \xi(a)=\xi(\bar{a})=(14) \\
& \xi(b)=\xi(c)=(12)(34) \\
& \xi(\bar{b})=\xi(\bar{c})=(13)(24) \\
& \xi(d)=\xi(\bar{d})=(23)
\end{aligned}
$$


where $a, \bar{a}, b, \bar{b}, c, \bar{c}, d$, and $\bar{d}$ are loops on $C P_{1}-\Sigma$ which are based at 0 and go once around the corresponding point $A, \bar{A}, B, \bar{B}, C, \bar{C}, D$, and $\bar{D}$ respectively, and avoid the rest of the points. See Ahara [1, Section 4].

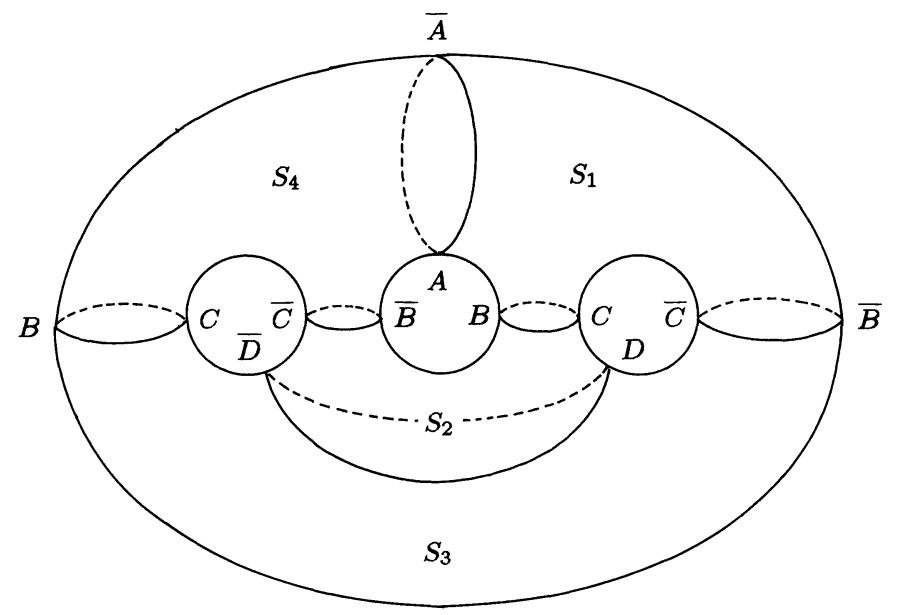

Figure 3

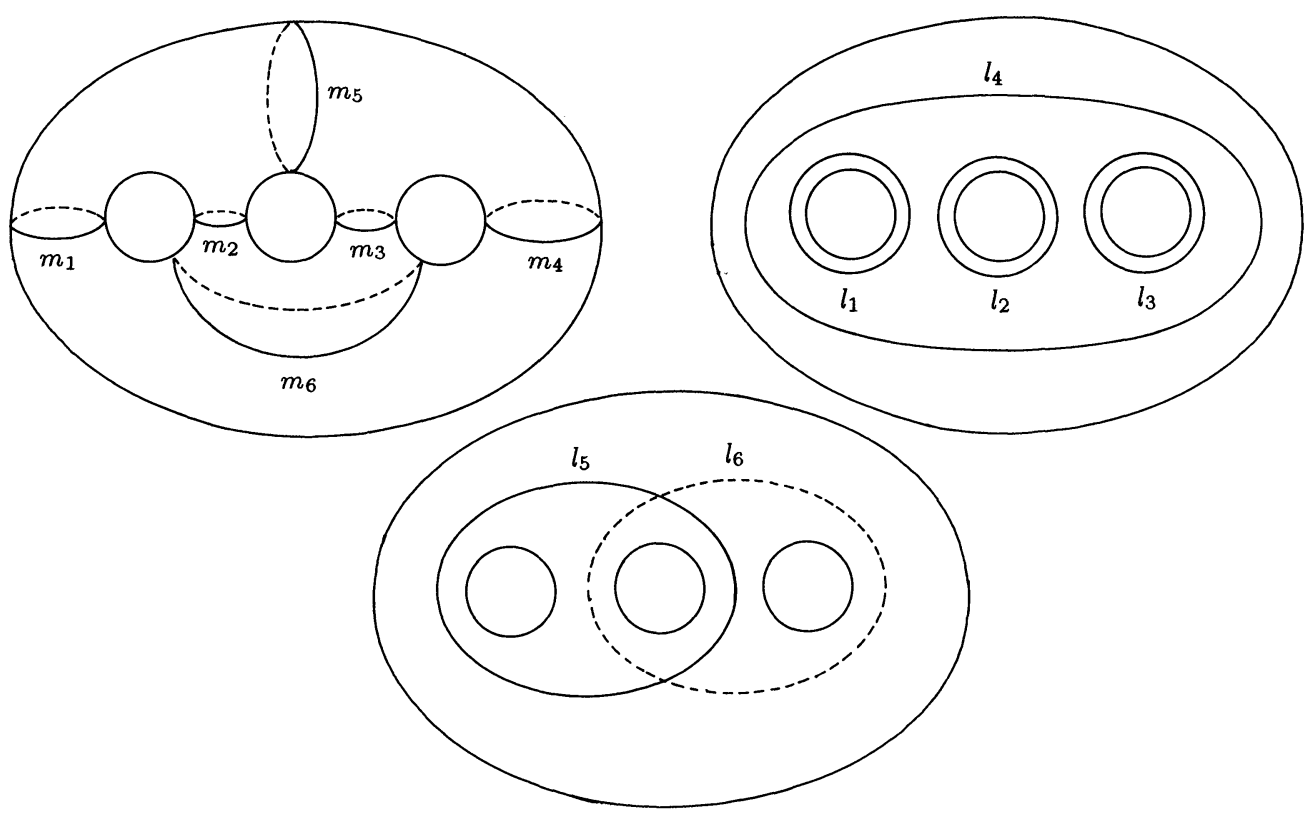

Figure 4

By the above facts,we can identify $F_{\sigma_{0}}$ as the surface constructed as follows: Take 4 copies of $C P_{1}$ and call them $S_{1}, S_{2}, S_{3}, S_{4}$, and cut slits open on them as shown in 
Figure 1.

Each of the resulting surfaces (again call $S_{i}, i=1,2,3,4$ ) is homeomorphic to a pair of pants. See Figure 2, in which the homeomorphism between $S_{3}$ and a pair of pants is given as a $180^{\circ}$ rotation of the slit $D \bar{D}$ followed by the natural identification. Similarly for $S_{4}$.

One obtains a surface of genus 3 by pasting these pairs of pants along their boundaries as shown in Figure 3. We will always identify $F_{\sigma_{0}}$ as the surface of Figure 3.

Now take twelve simple closed curves $\left\{m_{i}, l_{i}\right\}_{i=1,2, \cdots, 6}$ on $F_{\sigma_{0}}$ as shown in Figure 4.

Dehn twists about these simple closed curves are denoted by the corresponding capital letters. Thus $M_{1}$ for example denotes the positive Dehn twist about $m_{1}$, and $M_{1}^{-1}$ the negative twist. See Figure 5. Clearly, $M_{i} M_{j}=M_{j} M_{\imath}$ and $L_{i} L_{\jmath}=L_{\jmath} L_{\imath}$, for $i, j=1,2,3,4,5,6$.

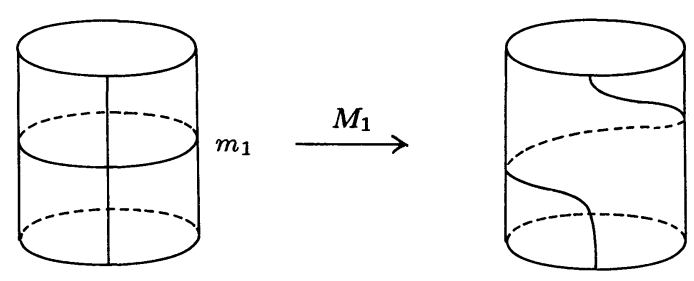

Figure 5

Let $\omega$ denote $\exp (2 \pi \sqrt{-1} / 5)$. Then the cyclic group $C_{5}=\left\{\omega^{2} \mid i=0,1,2,3,4\right\}$ acts on $V_{5}$ by $\omega^{2}:\left[z_{0}, z_{1}, z_{2}, z_{3}\right] \mapsto\left[\omega^{i} z_{0}, \omega^{2} z_{1}, z_{2}, z_{3}\right]$ and on $C P_{1}=C \cup\{\infty\}$ by the natural multiplication. Our fibration $f: V_{5} \rightarrow C P_{1}$ is equivariant with respect to the actions of $C_{5}$. [1, Section1].

By the action of $C_{5}$, we can identify $F_{\sigma_{0}}$ with the fibers over $\omega^{i} \sigma_{0}, i=1,2,3,4$. Let $\gamma:[0,1] \rightarrow C P_{1}-S F$ be a path. Then there exists a continuous family of homeomorphisms $\left\{H_{t}: F_{\gamma(0)} \rightarrow F_{\gamma(t)}\right\}_{0 \leq t \leq 1}$ such that $H_{0}=i d$ of $F_{\gamma(0)}$. If the path $\gamma$ joins $\omega^{i} \sigma_{0}$ and $\omega^{\jmath} \sigma_{0}$, we can define a monodromy homeomorphism $\rho(\gamma): F_{\sigma_{0}} \rightarrow F_{\sigma_{0}}$ by setting

$$
\rho(\gamma):=h_{j}^{-1} \circ H_{1} \circ h_{i}
$$

where $h_{\imath}: F_{\sigma_{0}} \rightarrow F_{\omega^{2} \sigma_{0}}$ and $h_{\jmath}: F_{\sigma_{0}} \rightarrow F_{\omega^{\jmath} \sigma_{0}}$ are homeomorphisms which give the identification by the action of $C_{5}$. The homeomorphism $\rho(\gamma)$ is determined up to isotopy by the homotopy class of $\gamma$ (fixing the terminal points $\gamma(0)$ and $\gamma(1)$ ).

Ahara [1, Section5] takes four paths $\gamma_{0}, \gamma_{1}, \gamma_{2}, \gamma_{3}$ joining $\sigma_{0}$ and $\omega \sigma_{0}$, whose homotopy classes are depicted in Figure 6. Any loop in $C P_{1}-S F$ based at $\sigma_{0}$ is homotopic to a composition of paths from the set of 20 paths

$$
\left\{\omega^{i} \gamma_{j}\right\}_{i=0 \cdots 4, \jmath=0 \cdots 3}
$$

where $\omega^{2} \gamma_{j}$ denotes the path $\gamma_{j}:[0,1] \rightarrow C P_{1}-S F$ rotated by the action of $\omega^{i}$ : $C P_{1} \rightarrow C P_{1}$. Clearly, $\omega^{3} \gamma_{j}$ joins $\omega^{i} \sigma_{0}$ and $\omega^{i+1} \sigma_{0}$. The monodromy representation $\rho$ : $\pi_{1}\left(C P_{1}-S F, \sigma_{0}\right) \rightarrow \mathcal{M}_{3}$ is completely determined if we give the homeomorphisms $\left\{\rho\left(\omega^{2} \gamma_{j}\right): F_{\sigma_{0}} \rightarrow F_{\sigma_{0}}\right\}_{i=0, \cdots, 4, j=0, \cdots, 3}$. 


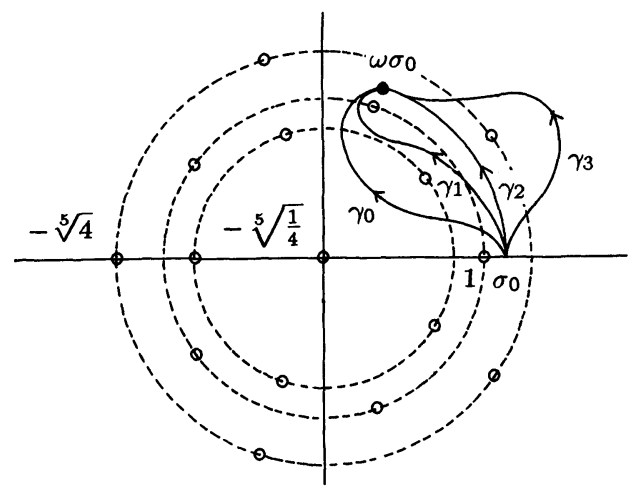

Figure 6

It is important to notice that the monodromy representation $\rho$ is an anti-homomorphism and that the monodromy homeomorphism associated with a composite path $\gamma \gamma^{\prime}$ ( $\gamma$ followed by $\gamma^{\prime}$ ) is computed by

$$
\rho\left(\gamma \gamma^{\prime}\right)=\rho\left(\gamma^{\prime}\right) \circ \rho(\gamma)
$$

Let $T: F_{\sigma_{0}} \rightarrow F_{\sigma_{0}}$ be an involution which is a $180^{\circ}$ rotation of $F_{\sigma_{0}}$ about the axis shown in Figure 7.

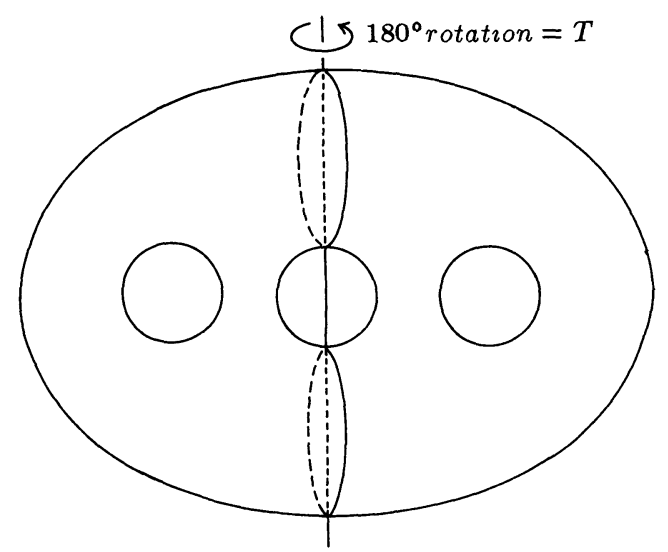

Figure 7

Now we are in a position to state our main result.

THEOREM 2.1. The monodromy homeomorphisms associated with $\omega^{\imath} \gamma_{j}(i=0,1$, $2,3,4, j=0,1,2,3)$ are independent of $i$, and are given as follows :

$$
\begin{aligned}
& \rho\left(\omega^{2} \gamma_{0}\right)=\rho\left(\gamma_{0}\right)=M_{1} M_{2} M_{3} M_{4} L_{1} L_{3} M_{6} L_{5} L_{6} T, \\
& \rho\left(\omega^{2} \gamma_{1}\right)=\rho\left(\gamma_{1}\right)=M_{1} M_{2} M_{3} M_{4} L_{1} L_{3} M_{6} T \\
& \rho\left(\omega^{2} \gamma_{2}\right)=\rho\left(\gamma_{2}\right)=M_{5}^{-1} L_{1} L_{3} M_{6} T \\
& \rho\left(\omega^{2} \gamma_{3}\right)=\rho\left(\gamma_{3}\right)=M_{5}^{-1} L_{1} L_{3} M_{6} L_{2}^{-1} L_{4}^{-1} T .
\end{aligned}
$$


Remarks. 1) In the statement of Theorem 2.1, and in what follows, we regard self-homeo-morphisms of $F_{\sigma_{0}}$ as elements of $\mathcal{M}_{3}$.

2) The involution $T$ commutes with $M_{1} M_{4}, M_{2} M_{3}, M_{5}, M_{6}, L_{1} L_{3}, L_{2}, L_{4}$, and $L_{5} L_{6}$.

3) Using Lemma 3.3 of Section 3, one can show

$$
\rho\left(\gamma_{0}\right)^{2}=T, \quad \rho\left(\gamma_{3}\right)^{2}=S T,
$$

where $S: F_{\sigma_{0}} \rightarrow F_{\sigma_{0}}$ is the involution whose construction is indicated by Figure 8. Note that $S T=T S$. It follows that $\rho\left(\gamma_{0}\right)$ and $\rho\left(\gamma_{3}\right)$ have order 4 in $\mathcal{M}_{3}$.

4) Let $Q \in \mathcal{M}_{3}$ be defined by

$$
Q=L_{1}^{-1} M_{4} L_{3} M_{3} L_{3} M_{4}
$$

Then using Lemma 3.3, one can show

$$
Q \rho\left(\gamma_{3}\right) Q^{-1}=\rho\left(\gamma_{0}\right)^{-1}
$$
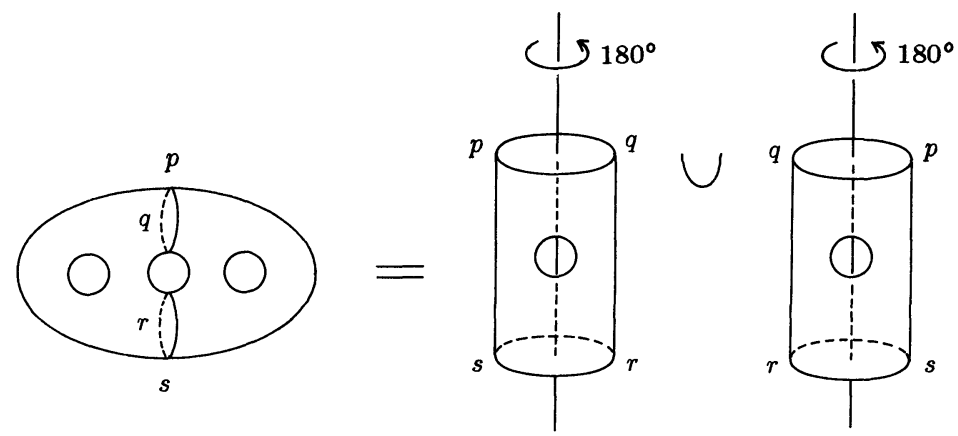

Figure 8

Here are some examples of monodromy calculations based on Theorem 2.1. The monodromy associated with loops $\gamma_{1} \gamma_{0}^{-1}, \gamma_{2} \gamma_{1}^{-1}, \gamma_{3} \gamma_{2}^{-1}$ (based at $\sigma_{0}$ ) are calculated as

$$
\begin{aligned}
& \rho\left(\gamma_{0}\right)^{-1} \rho\left(\gamma_{1}\right)=L_{5}^{-1} L_{6}^{-1} \\
& \rho\left(\gamma_{1}\right)^{-1} \rho\left(\gamma_{2}\right)=M_{6}^{-1} L_{3}^{-1} L_{1}^{-1}\left(M_{1}^{-1} M_{2}^{-1} M_{3}^{-1} M_{4}^{-1} M_{5}^{-1}\right) L_{1} L_{3} M_{6} \\
& \rho\left(\gamma_{2}\right)^{-1} \rho\left(\gamma_{3}\right)=L_{2}^{-1} L_{4}^{-1}
\end{aligned}
$$

respectively.

The monodromy around $F_{1}$ is given by

$$
\begin{aligned}
\rho\left(\left(\omega^{-1} \gamma_{1}\right)^{-1}\left(\omega^{-1} \gamma_{2}\right)\right) & =\rho\left(\gamma_{2}\right) \rho\left(\gamma_{1}\right)^{-1} \\
& =M_{1}^{-1} M_{2}^{-1} M_{3}^{-1} M_{4}^{-1} M_{5}^{-1},
\end{aligned}
$$

which coincides up to the sign convention for Dehn twists with the equation in the last example of [1, Section 5]. 
The monodromy around $F_{0}$ is given by

$$
\begin{aligned}
\rho\left(\gamma_{0}\left(\omega \gamma_{0}\right)\left(\omega^{2} \gamma_{0}\right)\left(\omega^{3} \gamma_{0}\right)\left(\omega^{4} \gamma_{0}\right)\right) & =\rho\left(\gamma_{0}\right)^{5}=\rho\left(\gamma_{0}\right) \\
& =M_{1} M_{2} M_{3} M_{4} L_{1} L_{3} M_{6} L_{5} L_{6} T,
\end{aligned}
$$

and the monodromy around $F_{\infty}$ is given by

$$
\begin{aligned}
\rho\left(\left(\omega^{4} \gamma_{3}\right)^{-1}\left(\omega^{3} \gamma_{3}\right)^{-1}\left(\omega^{2} \gamma_{3}\right)^{-1}\left(\omega \gamma_{3}\right)^{-1} \gamma_{3}^{-1}\right) & =\rho\left(\gamma_{3}\right)^{-5}=\rho\left(\gamma_{3}\right)^{-1} \\
& =T^{-1} L_{4} L_{2} M_{6}^{-1} L_{3}^{-1} L_{1}^{-1} M_{5}
\end{aligned}
$$

both of which have period 4 as noted in Remark 3 ).

Notice that these results also exemplify the correspondence between topological types of singular fibers and the monodromy homeomorphisms around them. See Theorem 1 of $[6]$.

\section{Outline of proof}

Let $B_{8}$ denote the braid group whose elements are isotopy classes of motions of the 8 points $p_{1}=\bar{D}, p_{2}=\bar{C}, p_{3}=\bar{B}, p_{4}=\bar{A}, p_{5}=A, p_{6}=B, p_{7}=C, p_{8}=D$ in $C P_{1}-\{0, \infty\}$. Following [1], we define elements $\beta_{i}, i=1, \cdots, 8$ of $B_{8}$ as in Figure 9 .
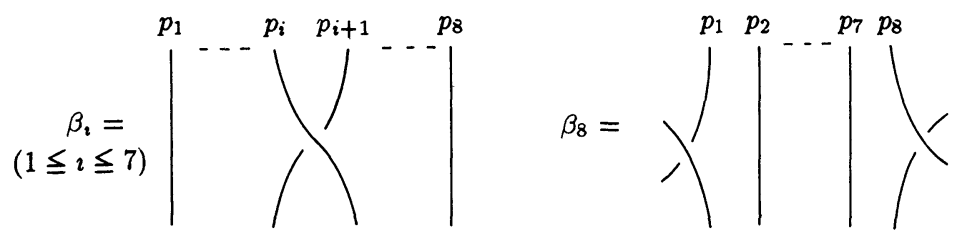

Figure 9

Proposition 3.1([1, Prop.5.3]). Suppose $\sigma$ moves along the path $\gamma_{i}(i=0,1,2$, or 3$)$. Then the corresponding motion $\beta\left(\gamma_{i}\right)$ of the points $\left\{p_{1}, p_{2}, p_{3}, p_{4}, p_{5}, p_{6}, p_{7}, p_{8}\right\}$ is represented as follows:

$$
\begin{aligned}
& \beta\left(\gamma_{0}\right)=\beta_{1}^{-1} \beta_{2} \beta_{1} \beta_{3}^{-1} \beta_{2}^{-1} \beta_{7}^{-1} \beta_{6} \beta_{7} \beta_{5}^{-1} \beta_{6}^{-1} \beta_{8} \beta_{4}^{-1} \beta_{1} \beta_{7} \\
& \beta\left(\gamma_{1}\right)=\beta_{1}^{-2} \beta_{3}^{-1} \beta_{5}^{-1} \beta_{4}^{-1} \beta_{3}^{-1} \beta_{5}^{-1} \beta_{7}^{-2} \beta_{2}^{-1} \beta_{4}^{-1} \beta_{6}^{-1} \\
& \beta\left(\gamma_{2}\right)=\beta_{1}^{-2} \beta_{3}^{-1} \beta_{5}^{-1} \beta_{4}^{-1} \beta_{3}^{-1} \beta_{5}^{-1} \beta_{7}^{-2} \\
& \beta\left(\gamma_{3}\right)=\left(\beta_{1}^{-1} \beta_{3}^{-1} \beta_{5}^{-1} \beta_{7}^{-1}\right)^{2}
\end{aligned}
$$

Remarks. 1) The motion of the 8 points described by a product $\beta \beta^{\prime}$ is isotopic to the motion $\beta$ followed by $\beta^{\prime}$.

2) The motion $\beta_{i}$ with even $i$ is lifted to Dehn twist(s) of $F_{\sigma_{0}}$, but with odd $i$ it is not lifted to any self-homeomorphism of $F_{\sigma_{0}}$. This point makes Theorem 2.1 non-trivial.

3) As we remarked in Section 2, the homeomorphisms $\rho\left(\gamma_{0}\right)$ and $\rho\left(\gamma_{3}\right)$ have order 4 in $\mathcal{M}_{3}$, but $\beta\left(\gamma_{0}\right)$ and $\beta\left(\gamma_{3}\right)$ are not of finite order in $B_{8}$. 
Let $\left\{x_{1}, x_{2}, x_{3}, x_{4}\right\}$ be the preimage of 0 under the branced covering $F_{\sigma_{0}} \rightarrow C P_{1}$, $x_{i}$ being in the pair of pants $S_{i}$ (see Section 2). The monodromy homeomorphism $\rho\left(\gamma_{i}\right): F_{\sigma_{0}} \rightarrow F_{\sigma_{0}}$ can be taken so that they preserve $\left\{x_{1}, x_{2}, x_{3}, x_{4}\right\}$. Then they cause permutations $\pi\left(\gamma_{i}\right)$ of the indices $(1,2,3,4)$.

Proposotion $3.2\left(\left[1\right.\right.$, Prop.5.6]). The permutations $\pi\left(\gamma_{i}\right)$ are given as follows: $\pi\left(\gamma_{0}\right)=(14), \pi\left(\gamma_{1}\right)=\pi\left(\gamma_{2}\right)=\pi\left(\gamma_{3}\right)=(1243)$.

Recall that we took 12 simple closed curves $\left\{m_{i}, l_{i}\right\}_{i=1, \cdots, 6}$ on $F_{\sigma_{0}}$ in Section 2 . The curve $l_{1}$ projects onto a curve on $C P_{1}$ shown in Figure 10, where the numbers $2,3,4$ by the curve are the "sheet numbers "which indicate the pair of pants onto which the portion of the curve is lifted.

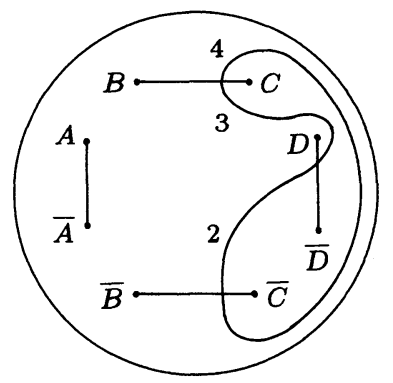

Figure 10

Applying the motion $\beta\left(\gamma_{0}\right)$ we move the projected curve, and as its final position we get a new curve. See Figure 11.

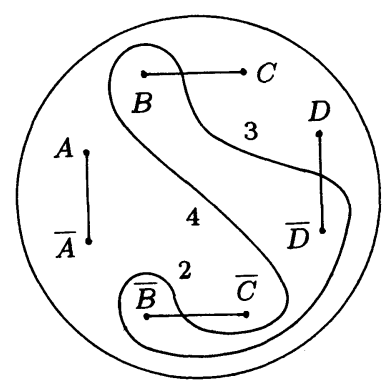

Figure 11

The sheet numbers attached to the new curves are determined by Proposition 3.2. The curve in Figure 11 is lifted to a curve on $F_{\sigma_{0}}$ as shown in Figure 12. 


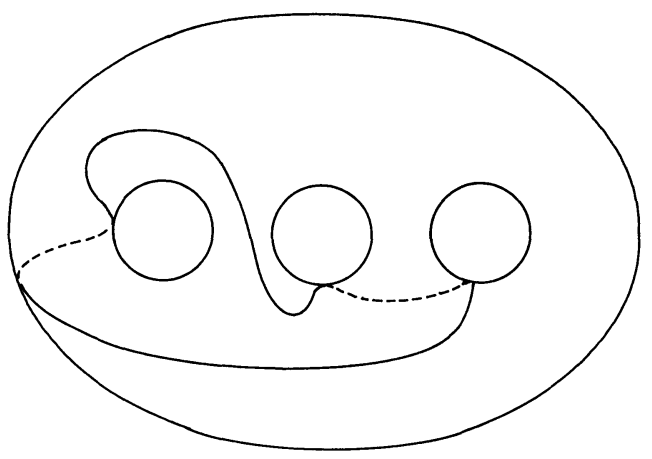

Figure 12

Therefore, the monodromy homeomorphism $\rho\left(\gamma_{0}\right)$ should send $l_{1}$ to the curve $\rho$ $\left(\gamma_{0}\right)\left(l_{1}\right)$ of Figure 12 . By the same method, we can draw the images $\rho\left(\gamma_{0}\right)\left(l_{2}\right), \rho\left(\gamma_{0}\right)\left(l_{3}\right)$, $\rho\left(\gamma_{0}\right)\left(m_{1}\right), \rho\left(\gamma_{0}\right)\left(m_{2}\right), \rho\left(\gamma_{0}\right)\left(m_{3}\right), \rho\left(\gamma_{0}\right)\left(m_{4}\right), \rho\left(\gamma_{0}\right)\left(m_{5}\right), \rho\left(\gamma_{0}\right)\left(m_{6}\right)$ as in Figure 13 .
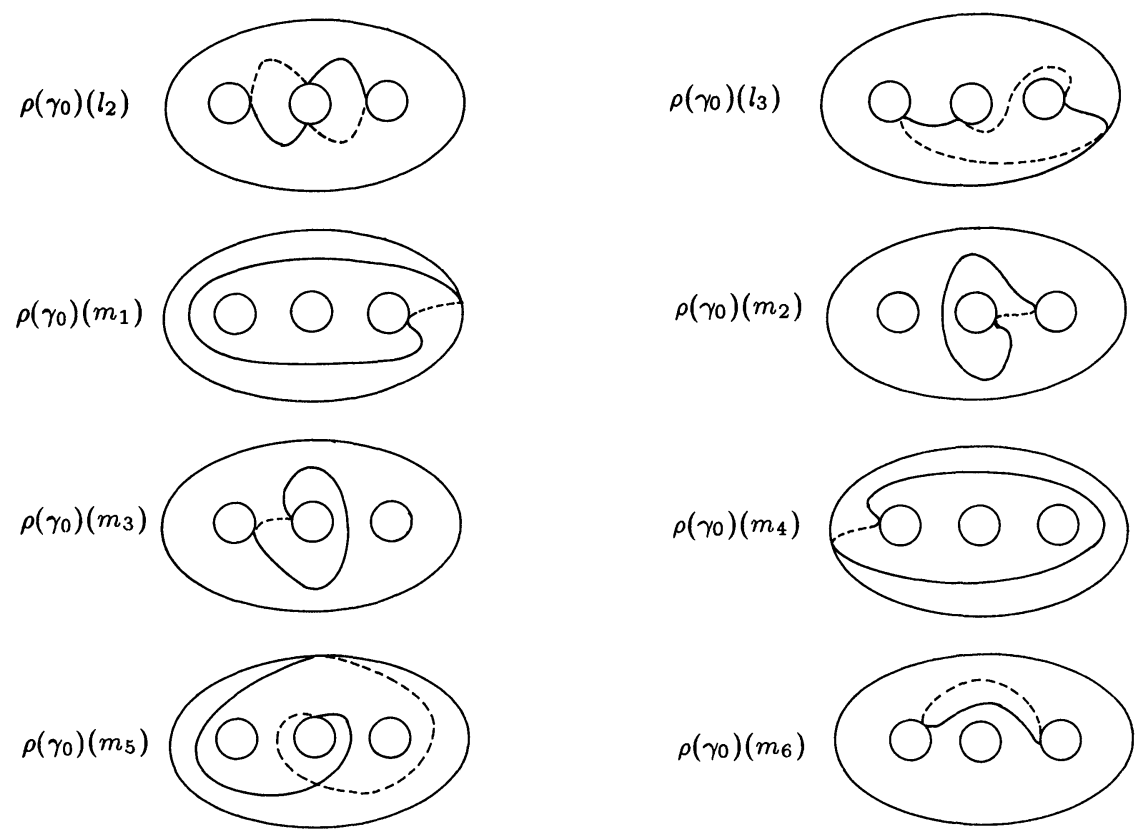

Figure 13

LEMMA 3.3. Let $h, h^{\prime}: F_{\sigma_{0}} \rightarrow F_{\sigma_{0}}$ be orientation preserving homeomorphisms. Suppose for each curve $C$ in $\left\{l_{1}, l_{2}, l_{3}, m_{1}, m_{2}, m_{3}, m_{4}, m_{5}, m_{6}\right\}$ the image $h(C)$ is freely homotopic to $h^{\prime}(C)$. Then $h$ is isotopic to $h^{\prime}$. 
Observe that each component of the complement of the union of the curves $l_{1}, l_{2}$, $l_{3}, m_{1}, m_{2}, m_{3}, m_{4}, m_{5}, m_{6}$ is an open disk and that no point is common to 3 or more curves. Then to prove Lemma 3.3 one can follow the arguments in the proofs of Lemmas 2.6 and 2.7 of Casson-Bleiler [2].

The first equality in Theorem 2.1 is proved by checking that the homeomorphism on the right hand side has the same images of $l_{1}, l_{2}, l_{3}, m_{1}, m_{2}, m_{3}, m_{4}, m_{5}, m_{6}$ as $\rho\left(\gamma_{0}\right)$, and then applying Lemma 3.3. Similarly for the rest of the equalities.

The author found the products of Dehn twists on the right hand sides of these equalities simply by trial and error.

\section{REFERENCE}

[1] K. Ahara, On the topology of Fermat type surface of degree 5 and the numerical analysis of algebraic curves, Tokyo J. Math. 16 (1993), 321-340.

[2] A.J. Casson and S.A. Bleiler, Automorphisms of surfaces after Nielsen and Thurston, London Math. Soc. Student Texts 9, 1988.

[ 3 ] S.K. Donaldson, Polynomial invariants for smooth four-manifolds, Topology 29 (1990), 141-168.

[ 4 ] M.H. Freedman, The topology of four-dimensional manifolds, J. Diff. Geom. 17 (1982), 357-454.

[5] F. Hirzebruch, Topological methods in algebraic geometry, Springer-Verlag, Berlin-HeidelbergNew York, 1966.

[6] Y. Matsumoto and J.M. Montesinos-Amilibia, Pseudo-periodic homeomorphisms and degeneration of Riemann surfaces, Bull. Amer. Math. Soc. 30 (1994), 70-75.

[ 7 ] J. Nielsen, Untersuchungen zur Topologie der geschlossenen zweiseitigen Flächen, Acta Math. 50 (1927), 189-358. English Translation by J.Stillwell: Investigations in the Topology of Closed Orientable Surfaces, Collected Math. Papers 1, Birkhäuser. (1986).

Department of Mathematical Sciences

UNIVERSITY OF TOKYO

HONGO,TOKYO 113 , JAPAN 